Supporting information

\title{
Additive manufacturing of shape memory polymer composites for futuristic technology
}

Jigar Patadiya ${ }^{a}$, Adwait Gawande ${ }^{b}$, Ganapati Joshi ${ }^{b}$, Balasubramanian Kandasubramanian ${ }^{a^{*}}$

a Rapid Prototyping Laboratory, Department of Metallurgical and Materials Engineering,

Defence Institute of Advanced Technology (DU), Ministry of Defence, Girinagar, Pune, India.

${ }^{b}$ Department of Aerospace Engineering, Defence Institute of Advanced Technology (DU), Ministry of Defence, Girinagar, Pune, India.

${ }^{*}$ Corresponding Author E-mail: meetkbs@gmail.com (B. Kandasubramanian)

Comparative image of two versatile and modern technology knows as 3D printing and 4D printing. Table contain list of current research on 4D printing of shape memory polymer composite with application. 


\begin{tabular}{|c|c|c|}
\hline & 3D Printing & 4D Printing \\
\hline Design & $\begin{array}{l}\text { CAD file containing digital } \\
\text { information of } 3 D \text { static part }\end{array}$ & $\begin{array}{l}\text { CAD file containing digital } \\
\text { information of } 3 D \text { astatic } \\
\text { part }\end{array}$ \\
\hline $\begin{array}{l}\text { Types of Material } \\
\text { used }\end{array}$ & Polymers, Metals, Ceramics, etc. & $\begin{array}{l}\text { Shape Memory Polymers, } \\
\text { Shape Memory alloys, } \\
\text { Shape Memory ceramics, } \\
\text { Shape Memory hybrids, } \\
\text { meta materials, hydrogels, } \\
\text { Multi materials, etc. }\end{array}$ \\
\hline $\begin{array}{l}\text { Nature of Printed } \\
\text { Structure }\end{array}$ & Static Structure & $\begin{array}{l}\text { Astatic or Dynamic } \\
\text { Structure }\end{array}$ \\
\hline Applications & $\begin{array}{l}\text { Engines, Launch vehicles, } \\
\text { Aircrafts, Technology } \\
\text { Demonstrators, etc. }\end{array}$ & $\begin{array}{l}\text { Deployable Structures: } \\
\text { Solar panel, hinges, } \\
\text { Antennas, Booms, } \\
\text { Collapsible Structures, ; } \\
\text { Morphing Wings, grippers, } \\
\text { etc. }\end{array}$ \\
\hline Stimuli Response & No & Yes \\
\hline
\end{tabular}

Figure S1: Differences in emerging 4D and 3D methodologies of printing.

Table S1: 4D Printing of SMP composites for different applications.

\begin{tabular}{|c|c|c|c|}
\hline 4D Printing Method & SMPC material & Application & Author \\
\hline FDM & Polylactic acid(PLA) SMP & Stress absorbers & Barletta et al. ${ }^{1}$ \\
\hline DLP & Elastomer(Covalently & Patterned laminate & Chao Y. et al. ${ }^{2}$ \\
& bonded), high water content & structure & \\
& hydrogel & Composite strip & Zeng et al. ${ }^{3}$ \\
& Carbon fiber reinforced & Polylactic acid(PLA) & \\
\hline FDM & SMP, Polylactic acid(PLA), & Actuator & Pyo et al. ${ }^{4}$ \\
& shape memory alloy wire & \\
\hline
\end{tabular}




\begin{tabular}{|c|c|c|c|}
\hline FDM & $\begin{array}{l}\text { Multi-walled carbon } \\
\text { nanotubes (MWCNTs) } \\
\text { SMPC's }\end{array}$ & $\begin{array}{l}\text { Composite structure } \\
\text { for small deployable } \\
\text { Spacecraft }\end{array}$ & Herath et al. ${ }^{5}$ \\
\hline $\begin{array}{c}\text { Mask-Image- } \\
\text { Projection-based } \\
\text { Stereolithography(SLA) }\end{array}$ & polystyrene film, liquid resin & 3D shell structures & Daniel et al. ${ }^{6}$ \\
\hline FDM & $\begin{array}{c}\text { Acrylonitrile butadiene styrene } \\
\text { (ABS) and a polyurethane type } \\
\text { SMP }\end{array}$ & $\begin{array}{c}\text { Double-layered } \\
\text { hinge }\end{array}$ & Ye Kang et al. ${ }^{7}$ \\
\hline FDM & PLA, CNT & Actuator & Yang Liu. et al. ${ }^{8}$ \\
\hline SLA & RSMP & $\begin{array}{l}\text { Light-weighted } \\
\text { architecture }\end{array}$ & Ang li et al. ${ }^{9}$ \\
\hline DLP & $\begin{array}{c}\text { Acrylic acid } \\
\text { Crosslinked } \\
\text { with BPA ethoxylate } \\
\text { dimethlyacrylate }\end{array}$ & $\begin{array}{l}\text { Tunable, deployable, } \\
\text { and mechanically } \\
\text { tunable lightweight } \\
\text { meta-materials } \\
\text { composite strip }\end{array}$ & Chen Yang et al. ${ }^{10}$ \\
\hline
\end{tabular}

\section{References}

(1) Barletta, M.; Gisario, A.; Mehrpouya, M. 4D Printing of Shape Memory Polylactic Acid (PLA) Components: Investigating the Role of the Operational Parameters in Fused Deposition Modelling (FDM). J. Manuf. Process. 2021, 61, 473-480. https://doi.org/10.1016/j.jmapro.2020.11.036. 
(2) Yuan, C.; Wang, F.; Ge, Q. Multimaterial Direct 4D Printing of High Stiffness Structures with Large Bending Curvature. Extrem. Mech. Lett. 2021, 42. https://doi.org/10.1016/j.eml.2020.101122.

(3) Zeng, C.; Liu, L.; Bian, W.; Liu, Y.; Leng, J. 4D Printed Electro-Induced Continuous Carbon Fiber Reinforced Shape Memory Polymer Composites with Excellent Bending Resistance. Compos. Part B Eng. 2020, 194 (April), 108034.

https://doi.org/10.1016/j.compositesb.2020.108034.

(4) Sun, J.; Du, L.; Scarpa, F.; Liu, Y.; Leng, J. Morphing Wingtip Structure Based on Active Inflatable Honeycomb and Shape Memory Polymer Composite Skin: A Conceptual Work. Aerosp. Sci. Technol. 2021, 111, 106541. https://doi.org/10.1016/j.ast.2021.106541.

(5) Herath, M.; Islam, M.; Epaarachchi, J.; Zhang, F.; Leng, J. 4D Printed Shape Memory Polymer Composite Structures for Deployable Small Spacecrafts. In ASME 2019 Conference on Smart Materials, Adaptive Structures and Intelligent Systems, SMASIS 2019; American Society of Mechanical Engineers, 2019. https://doi.org/10.1115/SMASIS2019-5583.

(6) Deng, D.; Chen, Y. 4D Printing: Design and Fabrication of 3D Shell Structures With Curved Surfaces Using Controlled Self-Folding. In Volume 1: Processing; American Society of Mechanical Engineers, 2015. https://doi.org/10.1115/msec2015-9459.

(7) Kang, Y.; Kim, K.; Ju, J. Reconfigurable Compliant Cellular Material with Programmable Compliant Cellular Structure. ASME Int. Mech. Eng. Congr. Expo. Proc. 2015, 9-2015. https://doi.org/10.1115/IMECE2015-52572.

(8) Liu, Y.; Zhang, W.; Zhang, F.; Leng, J.; Pei, S.; Wang, L.; Jia, X.; Cotton, C.; Sun, B.; Chou, T. W. Microstructural Design for Enhanced Shape Memory Behavior of 4D 
Printed Composites Based on Carbon Nanotube/Polylactic Acid Filament. Compos. Sci. Technol. 2019, 181 (June), 107692.

https://doi.org/10.1016/j.compscitech.2019.107692.

(9) Li, A.; Challapalli, A.; Li, G. 4D Printing of Recyclable Lightweight Architectures Using High Recovery Stress Shape Memory Polymer. Sci. Rep. 2019, 9 (1), 1-13. https://doi.org/10.1038/s41598-019-44110-9.

(10) Yang, C.; Boorugu, M.; Dopp, A.; Ren, J.; Martin, R.; Han, D.; Choi, W.; Lee, H. 4D Printing Reconfigurable, Deployable and Mechanically Tunable Metamaterials. Mater. Horizons 2019, 6 (6), 1244-1250. https://doi.org/10.1039/C9MH00302A. 